\title{
RESEARCH
}

Open Access

\section{Conditioned medium from bone marrow- derived mesenchymal stem cells inhibits vascular calcification through blockade of the BMP2-Smad1/5/8 signaling pathway}

Shuangshuang Wang ${ }^{1}$, Siwang Hư ${ }^{2}$, Jian Wang ${ }^{1}$, Yahui Liu' ${ }^{1}$, Ruochi Zhao ${ }^{1}$, Maoqing Tong ${ }^{1}$, Hanbin Cui ${ }^{1}$, Nan $\mathrm{Wu}^{1}$ and Xiaomin Chen ${ }^{1 *}$

\begin{abstract}
Background: Arterial calcification is associated with cardiovascular disease as a complication of advanced atherosclerosis and is a significant contributor to cardiovascular morbidity and mortality. Osteoblastic differentiation of vascular smooth muscle cells (VSMCs) plays an important role in arterial calcification and is characterized by cellular necrosis, inflammation, and lipoprotein and phospholipid complexes, especially in atherosclerotic calcification. The conditioned medium from bone marrow-derived mesenchymal stem cells (MSC-CM) is well known as a rich source of autologous cytokines and is universally used for tissue regeneration in current clinical medicine. Here, we demonstrate that MSC-CM inhibits beta-glycerophosphate ( $\beta$-GP)-induced vascular calcification through blockade of the bone morphogenetic protein-2 (BMP2)-Smad1/5/8 signaling pathway.

Methods: VSMC calcification was induced by $\beta$-GP followed by treatment with MSC-CM. Mineral deposition was assessed by Alizarin Red S staining. Intracellular calcium content was determined colorimetrically by the 0 cresolphthalein complexone method and alkaline phosphatase (ALP) activity was measured by the para-nitrophenyl phosphate method. Expression of BMP2, BMPR1A, BMPR1B, BMPR2, msh homeobox 2 (Msx2), Runt-related transcription factor 2 (Runx2), and osteocalcin (OC), representative osteoblastic markers, was assessed using real-time polymerase chain reaction analysis while the protein expression of BMP2, Runx2, and phosphorylated Smad1/5/8 was detected by western blot analysis.
\end{abstract}

Results: Our data demonstrated that MSC-CM inhibits osteoblastic differentiation and mineralization of VSMCs as evidenced by decreased calcium content, ALP activity, and decreased expression of BMP-2, Runx2, Msx2, and OC. MSC-CM suppressed the expression of phosphorylated Smad1/5/8 and the B-GP-induced translocation from the cytoplasm to the nucleus. Further study demonstrated that human recombinant BMP-2 overcame the suppression of VSMC calcification by MSC-CM.

Conclusion: MSC-CM may act as a novel therapy for VSMC calcification by mediating the BMP2-Smad1/5/8 signaling pathway

Keywords: Vascular calcification, Conditioned medium from bone marrow-derived mesenchymal stem cells, BMP2-Smad1/5/8 signaling, Atherosclerosis

\footnotetext{
* Correspondence: cxmdoctor@126.com

'Department of Cardiology, Ningbo First Hospital, Ningbo 315000, China

Full list of author information is available at the end of the article
}

C The Author(s). 2018 Open Access This article is distributed under the terms of the Creative Commons Attribution 4.0 International License (http://creativecommons.org/licenses/by/4.0/), which permits unrestricted use, distribution, and reproduction in any medium, provided you give appropriate credit to the original author(s) and the source, provide a link to the Creative Commons license, and indicate if changes were made. The Creative Commons Public Domain Dedication waiver (http://creativecommons.org/publicdomain/zero/1.0/) applies to the data made available in this article, unless otherwise stated. 


\section{Background}

Vascular calcification is a common feature of coronary artery disease as a complication of advanced atherosclerosis and is highly associated with aging, chronic kidney disease, diabetes, hypertension, and dyslipidemia [1, 2]. Calcification is a common finding in human atherosclerotic lesions, and arterial calcification is generally classified into calcification within an atherosclerotic plaque. During atherosclerosis, calcification develops in the arterial intima. Whereas medial calcification is a hallmark of diabetes and chronic kidney disease, arterial calcification is clearly associated with the mortality risk in individuals with atherosclerosis and diabetes. It has been reported that a spotty pattern of calcification is associated with coronary unstable ruptured plaques in patients with acute myocardial infarction $[3,4]$.

Increasing evidence now supports that osteo/chondrocytic transformation of vascular smooth muscle cells (VSMCs) and their dedifferentiation from a contractile to a proliferative phenotype are important for the initiation and progression of vascular calcification [1-3]. It is not clear to what extent trans-differentiation of VSMCs contributes to the pathogenesis of vascular calcification in vivo, although many in-vitro studies have demonstrated that VSMCs can undergo osteogenic differentiation and calcification [5-7]. Unfortunately, there are currently no ideal therapies directed at the prevention or treatment of vascular calcification to combat this growing problem in clinical practice today.

Bone marrow-derived mesenchymal stem cells (MSCs) have received a lot of attention for their efficacy as a medical therapeutic strategy, and have been shown to be efficacious for a variety of diseases such as myocardial infarction, corneal wounds, and lung injury; their efficacy has, in part, been attributed to their anti-inflammatory properties [8-10]. The conditioned medium from MSCs (MSC-CM) is well known as a rich source of autologous cytokines and is universally used in current clinical medicine. Recent studies have provided compelling evidence that vascular calcification is associated with inflammatory status and is enhanced by inflammatory cytokines, especially in atherosclerosis and diabetes [11-13]. MSC-CM is known to have potential anti-inflammatory activity. However, the functional role of MSC-CM in vascular calcification has not yet been elucidated. In this article, we investigated the effect of MSC-CM on VSMC calcification and the underlying mechanism.

\section{Methods}

\section{Reagents, antibodies, and assay kits}

Mouse MSCs (C57BL/6 mouse background) were purchased from Cyagen Biosciences (Guangzhou, China) and mouse VSMCs (C57BL/6 mouse background) were purchased from Procell Life Science \& Technology (Wuhan, China). Beta-glycerophosphate ( $\beta-G P$, G9422) and Alizarin Red S (A5533) were obtained from Sigma. Antibodies for BMP-2 (ab6285), Runx2 (ab76956), and p-Smad1/5/8 (ab66737) were purchased from Abcam. rhBMP-2 (B3555) and Noggin were purchased from Cyagen Biosciences. The Calcium Colorimetric Assay was obtained from Biovision, USA, and LabAssay ALP was purchased from Wako Pure Chemical Industries, Japan. The BCA protein assay was obtained from Pierce, USA.

\section{Cell culture}

MSCs and VSMCs were routinely cultured in complete medium containing Dulbecco's Modified Eagle's Medium (DMEM) with $4500 \mathrm{mg} / \mathrm{L}$ D-glucose, $4.00 \mathrm{nM} \mathrm{L-gluta-}$ mine, and sodium pyruvate supplemented with $10 \%$ FBS, $100 \mathrm{U} / \mathrm{ml}$ of penicillin and $100 \mu \mathrm{g} / \mathrm{ml}$ of streptomycin. The cells were incubated at $37{ }^{\circ} \mathrm{C}$ in a humidified atmosphere with $5 \% \mathrm{CO}_{2}$ and passaged at confluence with $0.5 \%$ trypsin/EDTA. VSMCs between passages 8 and 11 were used in this study.

The MSC-CM was collected every other day, and the suspended cells and debris were removed by centrifuging at $800 \times g$ for $10 \mathrm{~min}$ and filtering through a $50-\mu \mathrm{m}$ mesh and stored at $-80{ }^{\circ} \mathrm{C}$ until further use.

VSMCs were treated with DMEM medium (NC group), DMEM medium containing $10 \mathrm{mM} \beta-\mathrm{GP}\left(\mathrm{DMEM}^{\beta-\mathrm{GP}}\right.$ group), or MSC-CM with $10 \mathrm{mM} \beta$-GP (MSC-CM ${ }^{\beta-G P}$ group). The medium was changed every 2 days.

\section{Alizarin Red staining and quantification}

Mineral deposition in cultured VSMCs was assessed by Alizarin Red staining. Calcified VSMCs were fixed in 95\% alcohol for $15 \mathrm{~min}$ and exposed to $2 \%$ Alizarin Red ( $\mathrm{pH} 4.2$ ) for $5 \mathrm{~min}$ at room temperature. Cells were washed with deionized water to remove excess dye and images were taken using an inverted microscope. For Alizarin Red staining quantification, 10\% formic acid was used to elute Alizarin Red dye and the absorbance at $405 \mathrm{~nm}$ was determined with a microplate reader. Other plates of VSMCs treated with the same condition were used to normalize to protein content.

\section{Intracellular calcium content}

For quantification of calcium content, cells were washed with PBS and decalcified with $0.6 \mathrm{mM} \mathrm{HCl}$ at $4{ }^{\circ} \mathrm{C}$ for $24 \mathrm{~h}$. Calcium released from the cell cultures into the supernatant was determined colorimetrically by the $o$-cresolphthalein complexone method using the Calcium Colorimetric Assay and normalized to protein content. The protein content was determined using BCA protein assay (Pierce, USA).

\section{Alkaline phosphatase activity assay}

ALP activity of VSMCs was measured using LabAssay ALP (Wako Pure Chemical Industries), according to the 
manufacturer's protocol. ALP activity was normalized to total protein determined by BCA protein assay.

Reverse transcription PCR and real-time quantitative PCR To examine whether MSC-CM inhibits osteoblastic phenotype formation, the expression of BMP2, BMPR1A, BMPR1B, BMPR2, Msx2, Runx-2, and osteocalcin, representative osteoblastic markers, was assessed using real time-polymerase chain reaction analysis. Total RNA was isolated from cultured VSMCs using TRIzol Reagent (Invitrogen Corp, Carlsbad, CA, USA) according to the manufacturer's instructions. Reverse transcription was performed using an RT-PCR kit (Takara Biotech). Levels of mRNA were quantitated using an ABI 7500HT Fast Real-Time PCR System (Applied Biosystems, Grand Island, NY, USA). Glyceraldehyde-3-phosphate dehydrogenase (GAPDH) was used as an endogenous control. The $2^{-\Delta \Delta C t}$ method was used to calculate relative expression levels. Sequence-specific primers for the genes were designed using Premier 5 software and are presented in Table 1.

\section{Western blotting}

Cells were harvested and centrifuged at $1000 \times g$ at $4{ }^{\circ} \mathrm{C}$ for $5 \mathrm{~min}$, after which the supernatant was discarded and the samples were prepared using lysis buffer with protease inhibitors (1 $\mathrm{mmol} / \mathrm{L}$ benzamidine, $1 \mu \mathrm{g} / \mathrm{ml}$ leupeptin, $10 \mu \mathrm{g} / \mathrm{ml}$ soybean trypsin inhibitor, and $0.5 \mathrm{mmol} / \mathrm{L}$ PMSF). Samples were centrifuged at $14,000 \times g$ for $5 \mathrm{~min}$ and the supernatant was collected. The total protein

Table 1 Sequence-specific primers

\begin{tabular}{|c|c|c|}
\hline Gene & & Sequence $\left(5^{\prime}-3^{\prime}\right)$ \\
\hline \multirow[t]{2}{*}{$\overline{B M P 2}$} & Forward & TCTTCCGGGAACAGATACAGG \\
\hline & Reverse & TGGTGTCCAATAGTCTGGTCA \\
\hline \multirow[t]{2}{*}{ BMPR1A } & Forward & GGCCATTGCTTTGCCATTATAG \\
\hline & Reverse & CTTTCGGTGAATCCTTGCATTG \\
\hline \multirow[t]{2}{*}{ BMPRIB } & Forward & CCGACCTCGGTACAGCATTG \\
\hline & Reverse & GCTCTGAGACTGCTCGATCAAG \\
\hline \multirow[t]{2}{*}{ BMPR2 } & Forward & TTGGGATAGGTGAGAGTCGAAT \\
\hline & Reverse & TGTTTCACAAGATTGATGTCCCC \\
\hline \multirow[t]{2}{*}{ Ms $\times 2$} & Forward & GGAGCACCGTGGATACAGG \\
\hline & Reverse & TAGAAGCTGGGATGTGGTGAA \\
\hline \multirow[t]{2}{*}{ Runx2 } & Forward & ATGCTTCATTCGCCTCACAAA \\
\hline & Reverse & GCACTCACTGACTCGGTTGG \\
\hline \multirow[t]{2}{*}{ osteocalcin } & Forward & TGTCTTCTCCACAGCCTTCATG \\
\hline & Reverse & ACCACTCCAGCACAACTCCTTC \\
\hline \multirow[t]{2}{*}{ GAPDH } & Forward & ACCACAGTCCATGCCATCAC \\
\hline & Reverse & TCCACCACCCTGTTGCTGTA \\
\hline
\end{tabular}

content of the samples was measured using the BCA method, and sample aliquots containing equal amounts of protein were boiled for $5 \mathrm{~min}$ in sample loading buffer and separated by sodium dodecylsulphate polyacrylamide gel electrophoresis (SDS-PAGE) followed by PVDF membrane transfer (Immobilon-P; Millipore). Membranes were subsequently blocked with $5 \%$ nonfat dry milk in TBS containing $0.1 \%$ Tween 20 (TBST). Next, PVDF membranes were incubated with antibodies for BMP-2, Runx2, p-Smad1/5/8, or anti- $\beta$-actin (1:3000 diluted in TBS-T) overnight at $4{ }^{\circ} \mathrm{C}$, followed by an appropriate secondary antibody (horseradish peroxidase-conjugated anti-goat $\mathrm{IgG}$ ) for $1 \mathrm{~h}$ at room temperature. The reaction was visualized by enhanced chemiluminescence (ECL)/enhanced chemiluminescent system (Amersham Biosciences). Band intensities were quantified by Image J software (Wayne Rasband, NIH)/a VersaDoc (BioRad) scanning system and densitometry values were normalized to that of $\beta$-actin.

\section{Statistical analysis}

Data were expressed as mean \pm standard deviation of three independent experiments. Comparisons of parameters among groups were made by one-way ANOVA, followed by a homogeneity of variance test. Differences were considered statistically significant when $P<0.05$.

\section{Results \\ $\beta$-GP induced calcium deposition in VSMCs in a dose-dependent manner}

The VSMC calcification model was established by culture in the medium supplemented with 10,20 , or $40 \mathrm{mM}$ $\beta$-GP. The results of the Alizarin Red staining and quantification showed that $\beta$-GP induced a dose-dependent increase in calcium deposition in VSMCs (Fig. 1a, b). Further, the calcium content showed a dose-dependent increase (Fig. 1c).

\section{MSC-CM inhibits $\beta$-GP-induced calcium deposition in VSMCs}

To determine the effect of the MSC-CM on VSMC calcification, we investigated the calcium deposition and calcium content in VSMCs. Compared with the NC group, $10 \mathrm{mM}$ $\beta$-GP induced a significant increased calcium deposition of VSMCs (DMEM ${ }^{\beta-G P}$ group), as determined by Alizarin Red staining and quantification. In contrast, MSC-CM almost totally suppressed $\beta$-GP-induced calcium deposition in VSMCs (MSC-CM ${ }^{\beta-G P}$ group) (Fig. 2a, b).

Next, to further confirm the inhibitory effect of MSC-CM on calcification, we performed intracellular calcium content analysis in VSMCs. As shown in Fig. 2c, the calcium content was highly elevated in the medium with $\beta$-GP compared with the normal medium $\left(\mathrm{DMEM}^{\beta-G P}\right.$ group vs NC group). In contrast, the calcium content was 


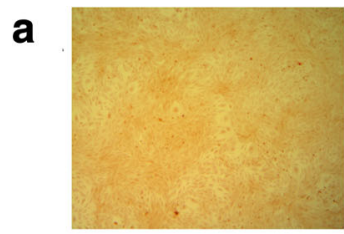

B-GP OmM

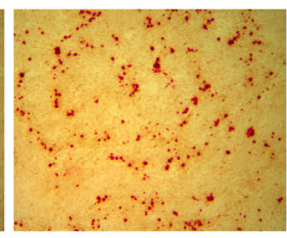

$10 \mathrm{mM}$

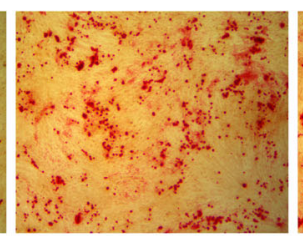

$20 \mathrm{mM}$

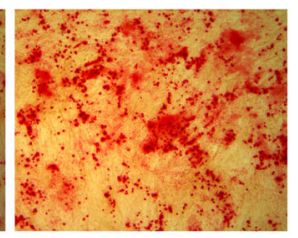

$40 \mathrm{mM}$
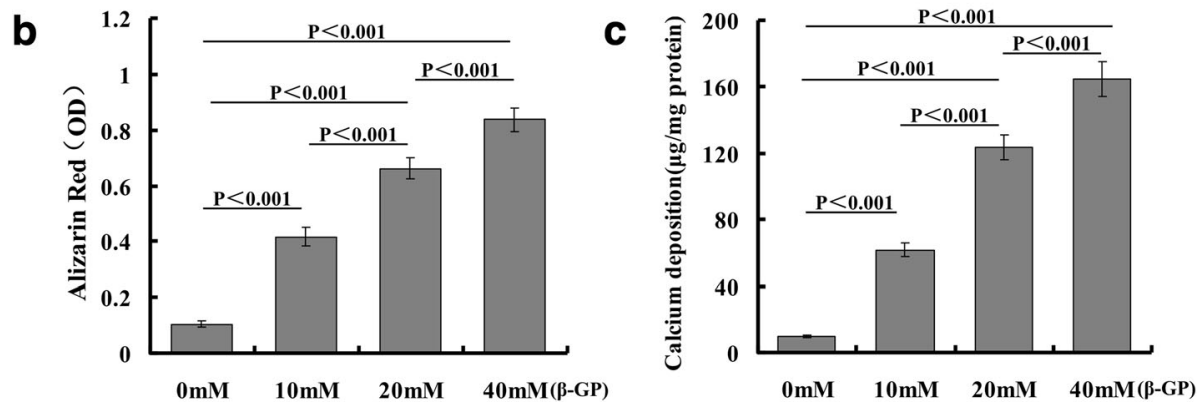

Fig. 1 B-GP induced calcium deposition in VSMCs in a dose-dependent manner. VSMCs cultured in normal medium or in calcification medium with 10, 20 and $40 \mathrm{mmol} / \mathrm{l}$ of $\beta-$ GP for 14 days. a Alizarin Red staining of microscopic views ( $\times 100)$. b Formic acid 10\% used to elute Alizarin Red dye and quantification of Alizarin Red staining measured with a microplate reader and normalized to total protein content. c Calcium content shown as mean \pm standard deviation of three independent experiments conducted in duplicate. $\beta$-GP beta-glycerophosphate, OD optical density

decreased obviously in the MSC-CM ${ }^{\beta-G P}$ group, compared to the $\mathrm{DMEM}^{\beta-\mathrm{GP}}$ group.

Taken together, these data indicate that MSC-CM treatment attenuated the osteoblastic differentiation and calcification of VSMCs. These results indicate that MSC-CM may be a novel therapeutic agent for VSMC calcification.

\section{MSC-CM abolished $\beta$-GP-induced ALP activity and expression of specific-osteogenic markers in VSMCs}

To further investigate the inhibitory effect of MSC-CM on VSMC calcification, we detected ALP activity and the expression of specific-osteogenic markers such as Runx2, Msx2, and osteocalcin in different VSMC groups. First, ALP activity was highly increased in the medium with $\beta$-GP compared with normal medium and this increase was significantly reduced by MSC-CM (Fig. 3a). Next, we observed that the expressions of Runx2, Msx2, and osteocalcin mRNA were increased when VSMCs were treated with $\beta$-GP. However, the increased mRNA expression of these markers was abolished by MSC-CM treatment (Fig. 3b-d). Runx2 protein expression was also demonstrably induced by $\beta$-GP and suppressed by MSC-CM (Fig. 3e, f).

\section{MSC-CM blocks BMP2-Smad1/5/8 signaling pathway}

We discovered that MSC-CM inhibited the BMP2 expression of VSMCs. In this experiment, we evaluated the mRNA and protein expression of BMP2 in cells treated with $\beta$-GP and/or MSC-CM. First, the expression of BMP2 mRNA and protein were increased when VSMCs were treated with $\beta$-GP. However, the increased mRNA and protein expression of BMP2 was suppressed by MSC-CM (Fig. 4a-c).

BMP2-Smad signaling is related to the BMP receptors and Smad transcription factors in osteogenesis [14]. The induction of BMP2 by treatment with $\beta$-GP and the inhibition of BMP2 by treatment with MSC-CM in VSMCs prompted us to investigate whether or not MSC-CM can repress the BMP receptor-Smad signaling pathway. Next, we investigated the expressions of BMP receptors in our model. As shown in Fig. 4d-f, BMPR1a, BMPR1b, and BMPR2 mRNA levels were induced 3 days after $\beta$-GP treatment. MSC-CM, on the other hand, inhibited the expression of BMPR1a, BMPR1b, and BMPR2 mRNA levels.

After the activation of BMP receptors, the expression and translocation of phosphorylated Smad1/5/8 from the cytoplasm to the nucleus may trigger osteoblast differentiation. Therefore, we examined whether MSC-CM regulated phospho-Smad1/5/8 expression in whole cells and nuclear and cytoplasm fractions, separately. We found that phospho-Smad1/5/8 expression was increased after $\beta$-GP treatment in VSMCs. The increase in phospho-Smad1/5/8 protein expression was completely suppressed by treatment with MSC-CM (Fig. 4g, h). Phosphorylated Smad1/5/8 in control VSMCs is normally expressed minimally in the nucleus but higher in 


\section{a}
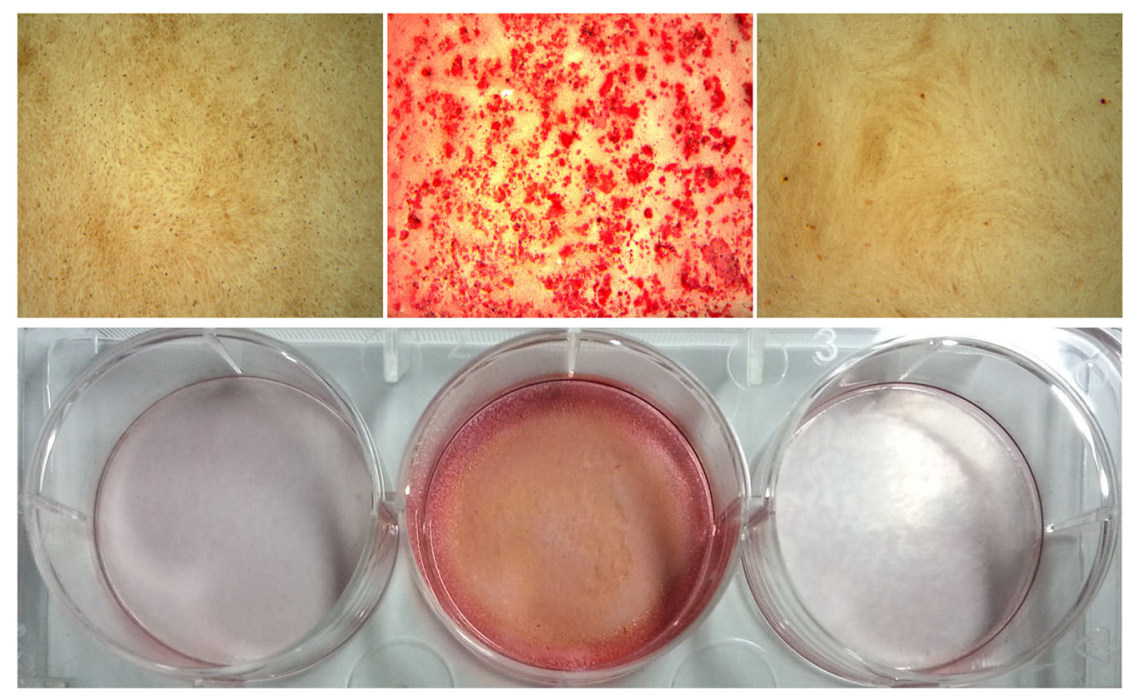

NC

DMEM ${ }^{\beta-G P}$

MSC-CM ${ }^{\beta-G P}$

b

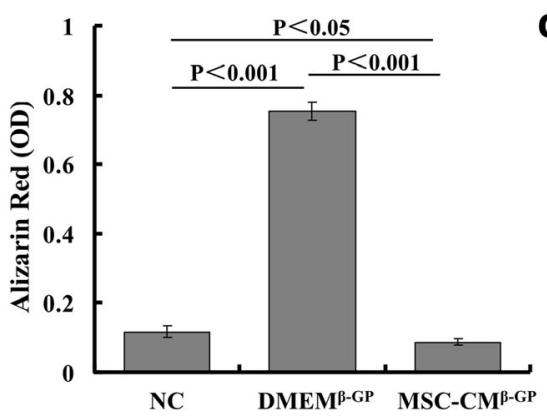

C

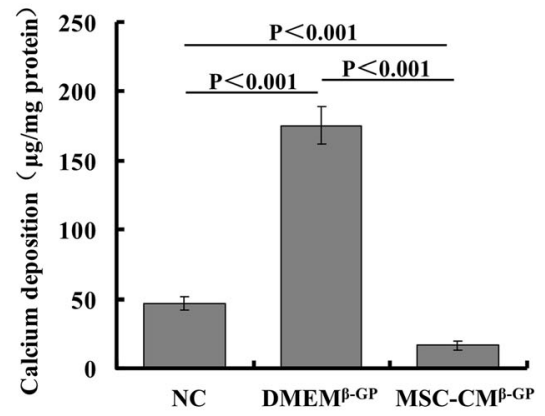

Fig. 2 MSC-CM inhibits $\beta$-GP-induced calcium deposition in VSMCs. Confluent cells incubated with normal medium or calcification medium with or without MSC-CM for 14 days. Calcification induced by addition of $\beta$-GP (10 mM). a Representative Alizarin Red staining of microscopic views ( $\times 100$, upper) and plates (lower). b Quantification of Alizarin Red staining. c Calcium contents measured and normalized by protein content of cell lysates. $\beta$-GP beta-glycerophosphate, DMEM Dulbecco's Modified Eagle's Medium, DMEM ${ }^{\beta-G P}$ DMEM medium containing 10 mM $\beta$-GP, MSCCM conditioned medium from bone marrow-derived mesenchymal stem cells, MSC-CM ${ }^{\beta-G P}$ MSC-CM with 10 mM $\beta-G P, N C$ DMEM medium, OD optical density

the cytoplasm. When VSMCs were stimulated by $\beta$-GP, however, phosphorylated Smad1/5/8 was mainly distributed in the nucleus, not in the cytoplasm. After treatment with MSC-CM, expression of phospho-Smad1/5/8 in the nucleus regressed while the cytoplasmic expression increased.

\section{BMP2 overcomes the inhibited effect of MSC-CM on $\beta$-GP-induced VSMC calcification}

To determine whether human recombinant BMP2 (rhBMP2) can reverse the inhibitory effect of MSC-CM on $\beta$-GP-induced calcification deposition, VSMCs were incubated with MSC-CM containing $200 \mathrm{ng} / \mathrm{ml}$ of rhBMP2. The concentrations of BMP-2 used were chosen based on previous study [5] and the potency of commercial preparations as indicated by the manufacturers. MSC-CM significantly decreased the calcium deposition and calcium content in VSMCs, which was overcome by rhBMP2 (Fig. $5 \mathrm{a}-\mathrm{c}$ ). Next, we found that
rhBMP2 upregulated the expression of Runx2, Msx2, and osteocalcin suppressed by MSC-CM (Fig. 5d-f). rhBMP2 also significantly increased Runx2, and phospho-Smad1/5/8 protein expression was inhibited by MSC-CM (Fig. 5g).

\section{Noggin, BMP2 antagonist, suppresses VSMC calcification by inhibiting Smad1/5/8 signaling}

We examined whether calcification induced by $\beta$-GP was mediated by BMP2. To test this hypothesis, the BMP antagonist, Noggin, was added to VSMCs prior to the addition of $\beta$-GP. VSMCs were pretreated with vehicle or Noggin $(100 \mathrm{ng} / \mathrm{ml})$ for $2 \mathrm{~h}$ followed by incubation with $\beta$-GP. Calcification, calcium deposition, osteoblast differentiation markers, and BMP2-Smad signaling were then investigated.

Noggin suppressed $\beta$-GP-induced calcium deposition in VSMCs, as determined by Alizarin Red staining and quantification (Fig. 6a, b). In addition, the increased 
a

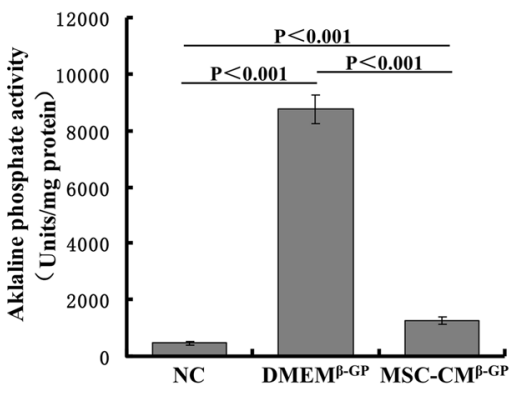

C

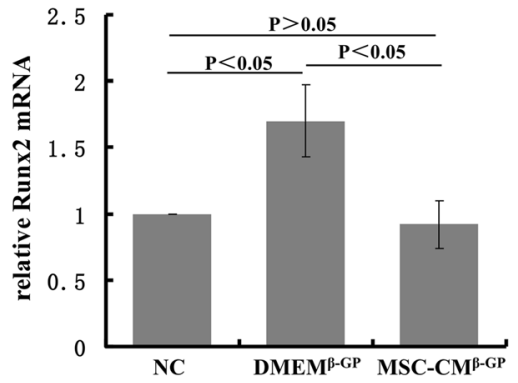

e

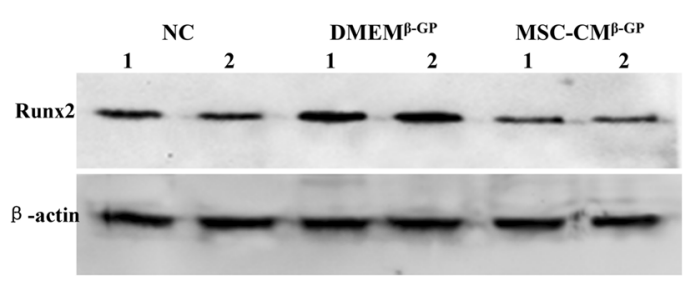

b
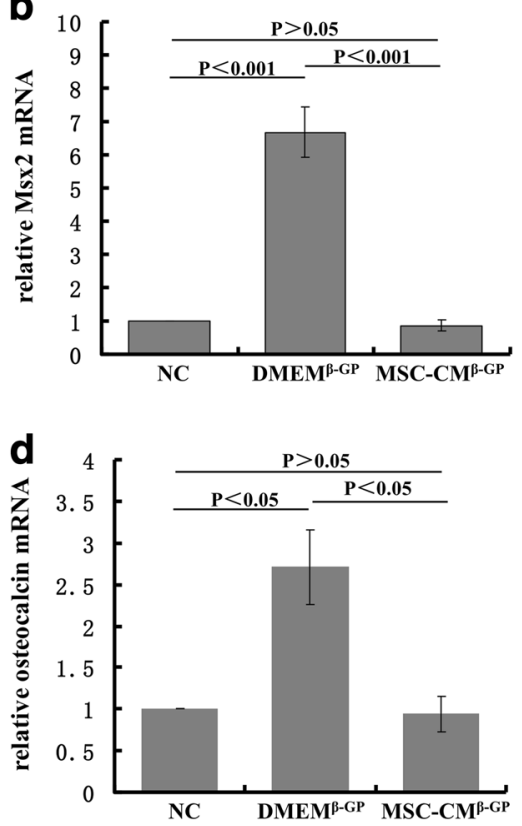

f

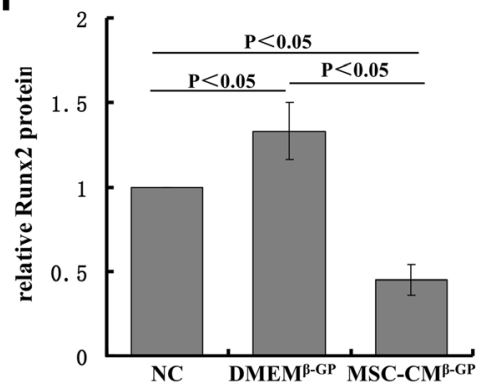

Fig. 3 MSC-CM abolished $\beta$-GP-induced ALP activity and expression of specific osteogenic markers in VSMCs. Confluent VSMCs incubated with different media in different groups. a ALP activity measured after 3 days by ALP kit, normalized to cellular protein contents. Levels of Ms×2 (b), Runx2 (c) and Osteocalcin (d) mRNA determined after 7 days by rt-PCR. e Representative immunoblot of Runx2. f Runx2 protein quantified by densitometry. $\beta-G P$ beta-glycerophosphate, DMEM Dulbecco's Modified Eagle's Medium, DMEM ${ }^{\beta-G P}$ DMEM medium containing 10 mM $\beta$-GP, MSC-CM conditioned medium from bone marrow-derived mesenchymal stem cells, MSC-CM ${ }^{\beta-G P}$ MSC-CM with 10 mM $\beta$-GP, Msx2 msh homeobox 2, NC DMEM medium, Runx2 Runt-related transcription factor 2

intracellular calcium content induced by $\beta$-GP was also inhibited by Noggin (Fig. 6c).

Quantitative real-time PCR demonstrated that Noggin blocked the $\beta$-GP-induced osteoblast differentiation markers BMP2, Runx2, Msx2, and osteocalcin in VSMCs (Fig. 6d-g). Western blot analysis similarly showed that Noggin decreases $\beta$-GP-induced BMP2 protein expression in VSMCs (Fig. 6h). Further, we investigated whether Noggin alters the expression of phospho-Smad1/5/8 protein in VSMCs. As predicted, the increased expression of phospho-Smad1/5/8 was blocked by treatment with Noggin (Fig. 6h).

\section{Discussion}

Vascular calcification is highly prevalent in many kinds of diseases including aging, atherosclerosis, diabetes, hypertension, chronic kidney disease, and dyslipidemia. In coronary arteries, calcium deposits influence atherosclerotic plaque stability and increase the incidence of acute coronary syndrome, depending on the size and distribution of the deposits [15]. In addition, the deposition of calcium minerals in the vessels, especially the medial arteries, ultimately results in vascular stiffening, increased pulse wave velocity, and increased pulse pressure, which therefore increases cardiac work, and eventually leads to left ventricular hypertrophy and diastolic dysfunction [15]. Vascular calcification is thus associated with a 3-fold to 4-fold increased risk for cardiovascular morbidity and mortality [4]. It resembles skeletal osteogenesis, and many bone cells as well as bone-related factors involved in both formation and resorption have been localized in calcified arteries. 


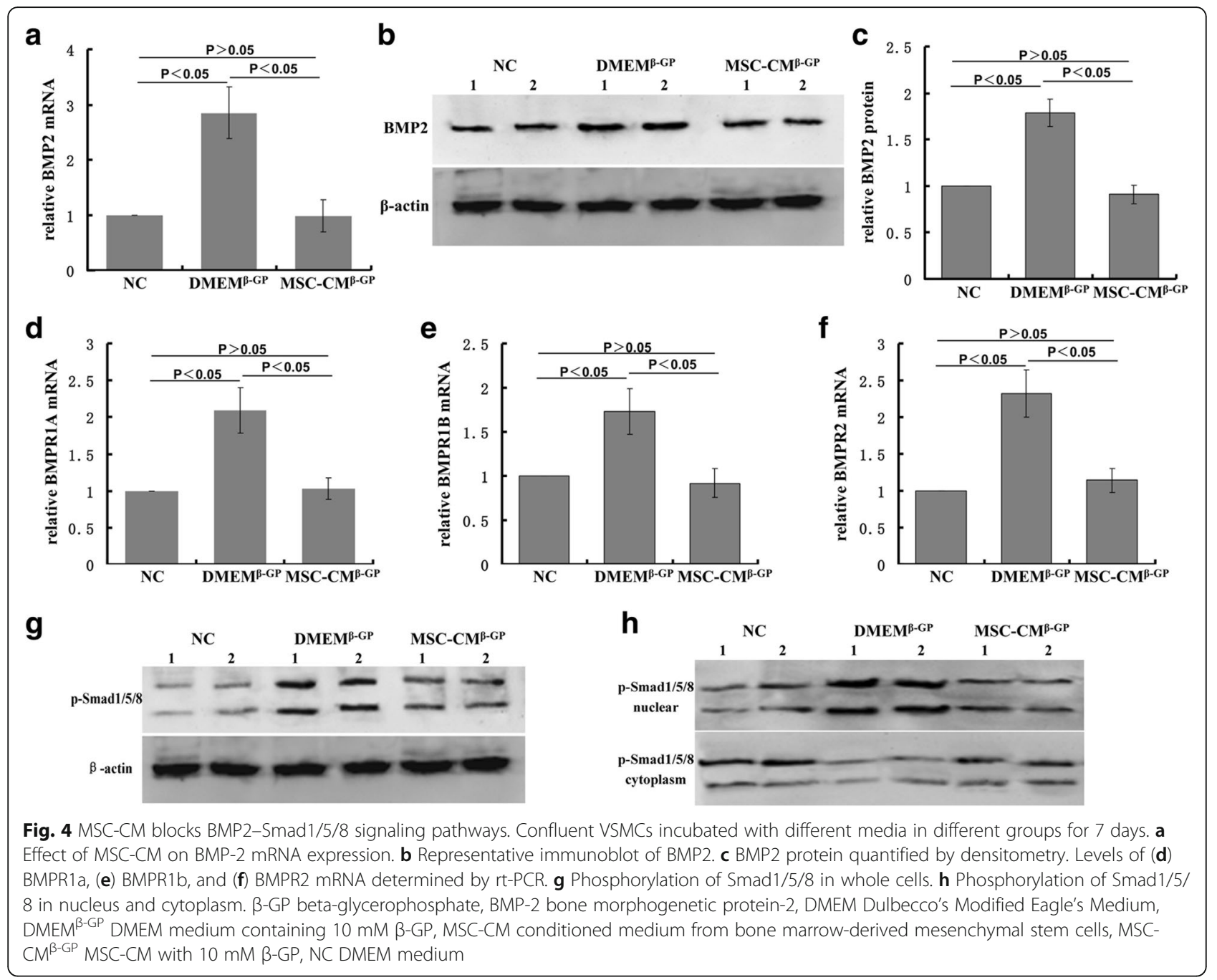

Bone marrow-derived mesenchymal stem cells (MSCs) have received a lot of attention for their efficacy as a medical therapeutic strategy and have been regarded as a future cell-based therapy. Recently, the therapeutic benefits of intravenous or local MSC transplantation have been observed in many diseases and injury models including acute lung injury $[10,16]$, myocardial infarction [17], acute renal failure [18], cerebral ischemia [19], Alzheimer's disease [20], and corneal damage [21]. However, low survival rates and the potential tumorigenicity of implanted MSCs could undermine the efficacy of cell-based therapy. The conditioned medium from MSCs (MSC-CM) is well known as a rich source of autologous cytokines. Therefore, the use of MSC-CM may be a feasible approach to overcome these limitations.

Recent studies have shown the usefulness of MSC-CM in many kinds of diseases in vitro or in vivo. For example, Timmers et al. [22] found that human MSC-CM improves cardiac function following myocardial infarction. Chen et al. [23] reported that MSC-CM prevents radiation-induced liver injury by inhibiting inflammation and protecting sinusoidal endothelial cells. Tamari et al. [24] additionally suggested that the MSC-CM, containing growth factor derived from stem cells, is able to accelerate wound healing. Finally, Kim et al. [25] found that production of PGE2 by MSCs and subsequent production of IL-10 were required to reduce the severity of colitis.

The data presented in this study suggest that MSC-CM is a potential therapeutic treatment for vascular calcification. To the best of our knowledge, this is the first demonstration that MSC-CM can prevent beta-glycerophosphate ( $\beta$-GP)-induced vascular smooth muscle cell (VSMC) calcification via downregulated BMP2 expression and blockade of the BMP2 signaling cascade. We found that MSC-CM inhibited calcification and osteoblast differentiation markers in cultured VSMCs.

In this study, we established an in-vitro model of VSMC calcification using $\beta$-GP. The calcification and calcium content showed a dose-dependent increase in the presence of 
a
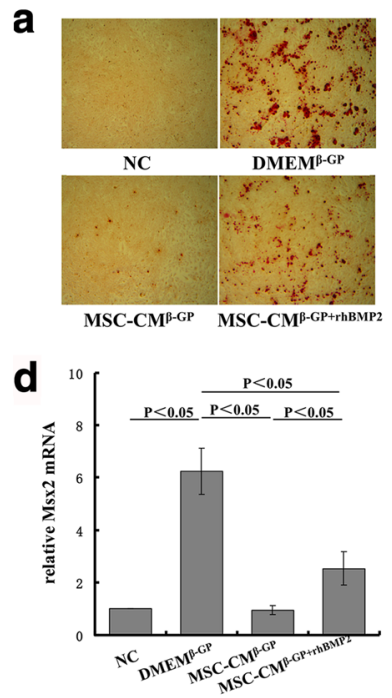

g

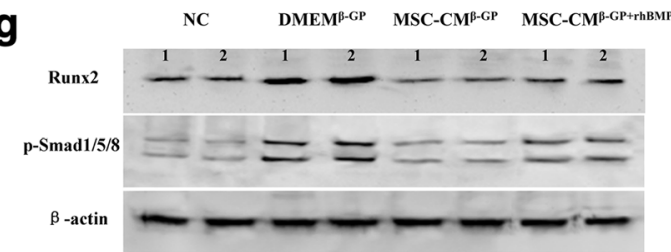

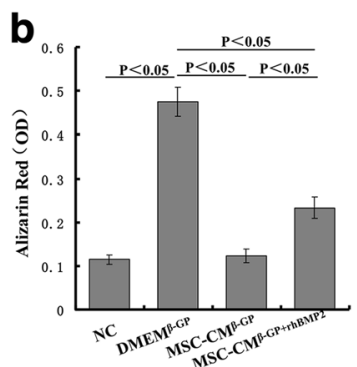
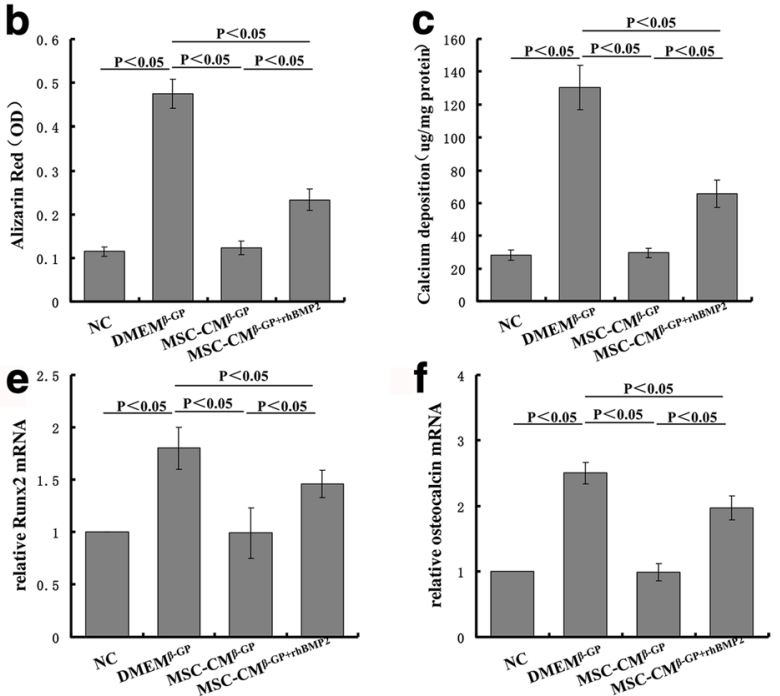

Fig. 5 BMP2 overcomes inhibited effect of MSC-CM on $\beta$-GP-induced VSMC calcification. a Microscopic views ( $\times 100$ ) of Alizarin Red staining. b Quantification of Alizarin Red staining. c Calcium contents measured and normalized by protein content of cell lysates. Expression of Msx2 (d), Runx2 (e), and osteocalcin (f) mRNA assessed by rt-PCR. g Cell lysates analyzed by western blot analysis with anti-Runx2 and p-Smad1/5/8. $\beta$-GP beta-glycerophosphate, DMEM Dulbecco's Modified Eagle's Medium, DMEM ${ }^{\beta-G P}$ DMEM medium containing 10 mM $\beta$-GP, MSC-CM conditioned medium from bone marrow-derived mesenchymal stem cells, MSC-CM ${ }^{\beta-G P}$ MSC-CM with 10 mM $\beta$-GP, Msx2 msh homeobox 2, NC DMEM medium, OD optical density, Runx2 Runt-related transcription factor 2

10,20 , and $40 \mathrm{mM}$ of $\beta-\mathrm{GP}$, a finding that has been verified by many researchers $[6,7,26]$. We found that MSC-CM dramatically inhibited $\beta$-GP-induced VSMC calcification and calcium deposition as determined by calcium amounts and Alizarin Red staining. To further investigate the inhibitory effect of MSC-CM on VSMC calcification, we detected alkaline phosphatase (ALP, an important enzyme in early osteogenesis) activity and specific osteogenic markers which were associated with the transition of the bone formation phenotype (i.e., Runt-related transcription factor 2 (Runx2), msh homeobox 2 (Msx2), and osteocalcin (OC)). Accumulating studies have demonstrated that vascular calcification is associated with the upregulation of these makers [27-30]. The results demonstrate that MSC-CM abolished $\beta$-GP-induced ALP activity and expression of osteogenic markers in VSMCs. These findings indicate that MSC-CM may be considered as a therapeutic agent to prevent or treat vascular calcification.

Some different signaling pathways work in vascular calcification, such as the BMP2-Smad1/5/8, NF-kBRANKL, Wnt- $\beta$-catenin, endothelin-1-endothelin receptor, and AMPK-mTOR pathways. In this article, we demonstrated that MSC-CM suppression of calcification may be mediated by expression of BMP2 and the BMP2 receptor-Smad1/5/8 signaling pathway. Bone morphogenetic proteins (BMPs) are a superfamily of transforming growth factor beta (TGF- $\beta$ ) and secretory growth factor, which are reported to have osteogenic actions and play important roles in bone formation. BMP-2 is an important molecule in the regulation of bone formation as well as vascular calcification. Previous studies have demonstrated that BMP-2 plays a crucial role in osteoblast differentiation in the development of atherosclerosis and calcific vascular lesions [31, 32]. In in-vivo models of arterial calcification, BMP-2 gene expression is upregulated in the aortic adventitia and atherosclerotic plaques [33]. In addition, BMP2 accelerated atherosclerotic intimal calcification in BMP2 transgenic/apoE knockout mice [34]. Blockage of BMP-2 action by the antagonist, Noggin, inhibits osteoblast differentiation and bone formation in vivo and in vitro [35]. BMP signaling has consequently been addressed as a therapeutic target in vascular diseases.

In this study, we assessed the inhibitory potential of MSC-CM regarding osteogenesis. The results demonstrated that VSMCs, when stimulated by $10 \mathrm{mM} \beta$-GP, 

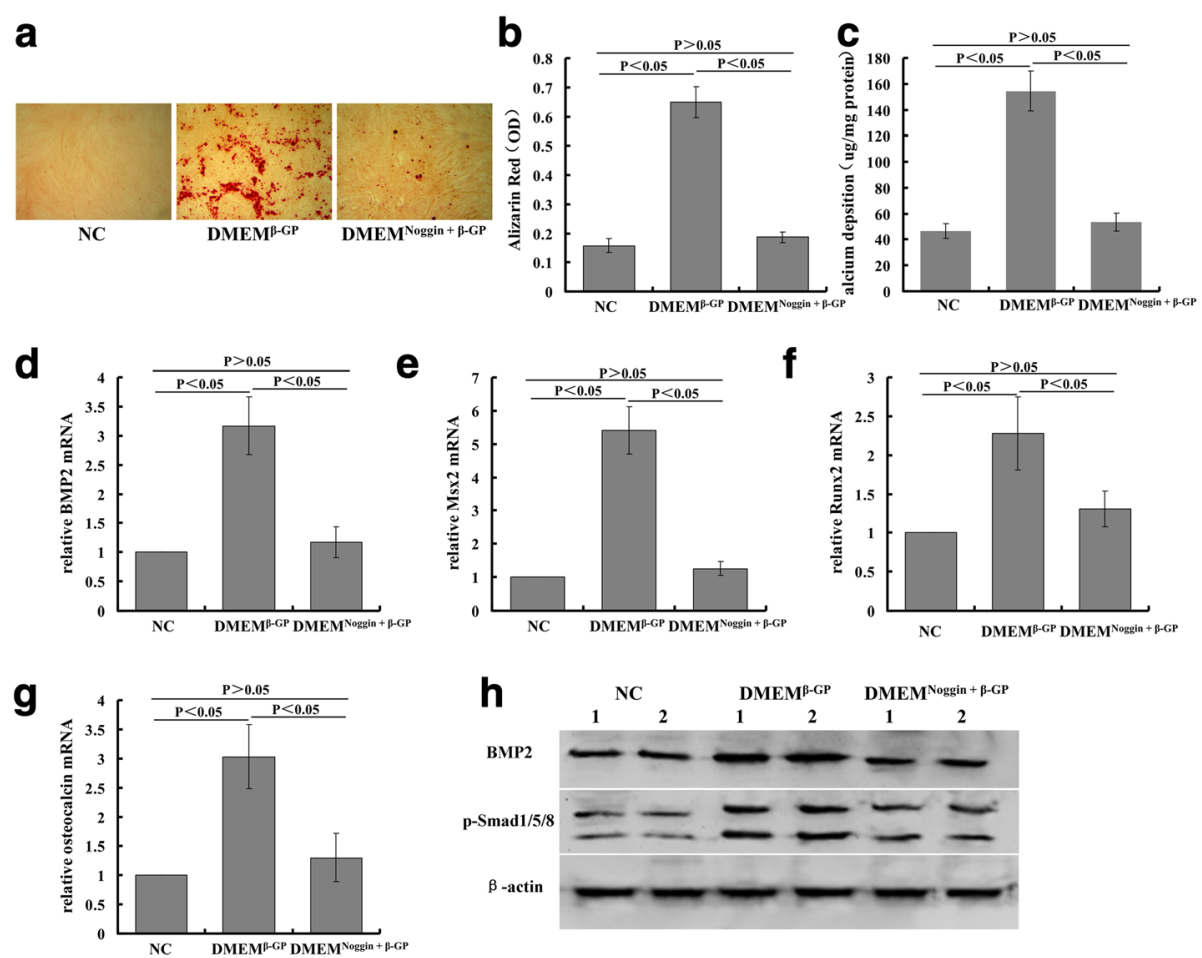

Fig. 6 Noggin, BMP2 antagonist, suppresses VSMC calcification by inhibiting Smad1/5/8 signaling. VSMCs pretreated with Noggin $(100 \mathrm{ng} / \mathrm{ml})$ or vehicle for $2 \mathrm{~h}$ followed by incubation with $10 \mathrm{mM} \beta$-GP. a Alizarin Red staining ( $\times 100)$. b Quantification of Alizarin Red staining. c Calcium contents measured and normalized by protein content of cell lysates. mRNA expression of (d) BMP2, (e) Msx2, (f) Runx2, and ( $\mathbf{g}$ ) osteocalcin analyzed by rt-PCR. $\mathbf{h}$ Protein expression of BMP2 and phospho-Smad1/5/8 performed by western blot analysis. $\beta-G P$ beta-glycerophosphate, DMEM Dulbecco's Modified Eagle's Medium, DMEM ${ }^{\beta-G P}$ DMEM medium containing 10 mM $\beta-G P$, DMEM ${ }^{\text {Noggin }}$ $+\beta-G P$ DMEM medium containing $10 \mathrm{mM} \beta-$ GP and $100 \mathrm{ng} / \mathrm{ml}$ Noggin, Msx2 msh homeobox 2, NC DMEM medium, OD optical density, Runx2 Runt-related transcription factor 2

resulted in increased BMP-2 expression. Conversely, this effect was inhibited by treatment with MSC-CM, suggesting that MSC-CM suppression of calcification is mediated by inhibiting the expression of BMP2.

Gene responses to BMPs are mediated by Smad transcription factors after being phosphorylated by the BMP receptor complex [36]. Upon binding of BMP2, the type-II receptor (BMPR-II) transphosphorylates the type-I receptor (BMPR-I) and the phosphorylated BMPR-I, in turn, phosphorylates a set of intracellular substrate signaling proteins collectively known as receptor-regulated Smads (R-Smads), Smad1, Smad5, and Smad8 [36-39]. Next, phosphorylated Smad1/5/8 dissociates from BMPR-I, forms a heteromeric complex with a common-partner Smad (Co-Smad), Smad4, and translocates from the cytoplasm into the nucleus where they regulate transcription of various target genes, including Runx2 and Msx2, to increase various kinds of osteogenic differentiation indicators $[40,41]$. Several studies support that the BMP2Smad1/5/8 signaling pathway has been involved in bone remodeling processes including osteoblast differentiation and osteoclastogenesis [42, 43]. Considering that BMP2 is closely associated with the Smad pathway, we hypothesized that the inhibitory effect of MSC-CM on VSMC calcification is mediated by Smad1/5/8 signaling. Therefore, we investigated whether the MSC-CM inhibition of calcification was related to the phospho-Smad1/5/8 signaling pathway.

In this study, when VSMCs were stimulated by $\beta$-GP, mRNA expression of BMPR-I and BMPR-II increased, although the increase was suppressed by MSC-CM. Western blot analysis further showed that phosphorylated Smad1/5/8 was markedly upregulated when induced by $\beta$-GP, and downregulated when treated with MSC-CM. Next we tested the expression of phosphorylated Smad1/ $5 / 8$ in the nucleus and cytoplasm separately. Western blot analysis showed that phosphorylated Smad1/5/8 in control VSMCs was low in nuclear regions and higher in the cytoplasm. When VSMCs were stimulated by $\beta$-GP, phosphorylated Smad1/5/8 mainly became distributed in the nucleus, not in the cytoplasm. These results suggested that $\beta$-GP not only promoted the expression of phosphorylated Smad1/5/8, but also accelerated its translocation from the cytoplasm to the nucleus. Treatment with MSC-CM, on the other hand, reduced the nuclear presence of phospho-Smad1/5/8 and increased its presence in the cytoplasm. Together, the results suggest that 
MSC-CM inhibited the expression of phosphorylated Smad1/5/8 and its transfer from the cytoplasm to the nucleus, where it plays a role in osteoblast differentiation.

To further investigate the role of BMP2 and the inhibitory effect of MSC-CM on VSMC calcification, we performed an experiment by which VSMCs were treated with MSC-CM in the presence of human recombinant BMP-2 (rhBMP2). Previous research has shown that BMP-2 enhances elevated phosphate-induced calcification, but does not induce calcification under normal phosphate conditions [44]. Our research also showed the same results: rhBMP2 strengthened $\beta$-GP-induced calcification and, in addition, both rhBMP2 in DMEM and that in MSC-CM did not cause calcification without $\beta$-GP (results not shown). When we added rhBMP2 in MSC-CM with $\beta$-GP, it overcame the suppression of MSC-CM on $\beta$-GP-induced VSMC calcification. We observed that rhBMP2 improved the calcium deposition and calcium content in the VSMCs which was inhibited by MSC-CM. rhBMP2 also upregulated several genes involved in osteoblastic transformation of VSMCs, which had been previously reduced by MSC-CM. rhBMP2 also significantly increased Runx 2 and phospho-Smad1/5/8 protein expression inhibited by MSC-CM. These results demonstrated that MSC-CM inhibited VSMC calcification by suppressing BMP2.

Next, we demonstrated that BMP2 is implicated in the regulation of calcification, using the BMP2 antagonist Noggin to show the relevance of BMP2 in VSMC calcification. Noggin was identified as a BMP4 antagonist as well as other BMPs including BMP2, BMP7, and BMP14. In this study, the results show that Noggin inhibited $\beta$-GP-induced VSMC calcification, calcium content, and the associated marker genes, such as BMP2, Msx2, Runx2, and osteocalcin, suggesting that BMP2 and its downstream signaling are involved in the process of VSMC calcification.

Although our study shows that MSC-CM inhibition of calcification may be mediated via the regulation of BMP2 expression, we did not know which active component secreted by MSC-CM is responsible for calcification inhibition. Many research studies showed that MSC-CM contains various cytokines, such as insulin-like growth factor (IGF)-1, vascular endothelial growth factor (VEGF), and interleukin (IL)-10, which may play crucial roles in treatment of multiple diseases. Our results also showed that MSC-CM contained conspicuous high levels of IGF $(74.4 \pm 1.2$ vs $0.09 \pm 0.01 \mathrm{pg} / \mathrm{ml})$, VEGF $(813.1 \pm 19.5$ vs $0.0 \pm 0.0 \mathrm{pg} / \mathrm{ml})$, and IL-10 $(98.9 \pm 2.7 \mathrm{vs} 6.9 \pm 2.3 \mathrm{pg} / \mathrm{ml})$ compared with the NC group, which may work in inhibiting VSMC calcification. However, the elucidation of the exact molecular mechanism responsible for cytoprotection can only be defined by another complex and long-term undertaking beyond the scope of this article.

\section{Conclusion}

This study is the first to demonstrate that MSC-CM exerts significant protective effects on VSMC calcification. MSC-CM elicits this protection through reduction of BMP2-dependent Smad1/5/8 signaling, which plays a pivotal role in vascular calcification. This study could potentially extend the repertoire of stem cell-based therapy, as the data suggest that secretions of stem cells may offer a viable alternative to circumvent the complications associated with clinical cell-based therapy such as immunocompatibility, tumorigenicity, ethics, costs, and waiting time for ex-vivo expansion. In order to investigate that application further, however, the components secreted by MSC-CM which are responsible for cytoprotection must be studied further. These findings are the first to implicate MSC-CM as a contributor of a protective effect against calcification in vitro and identify a novel potential therapeutic for vascular calcification and its associated diseases.

\section{Abbreviations \\ ALP: Alkaline phosphatase; BMP-2: Bone morphogenetic protein-2; DMEM: Dulbecco's Modified Eagle's Medium; IGF-1: Insulin-like growth factor- 1; IL-10: Interleukin-10; MSC: Mesenchymal stem cell; MSC-CM: Conditioned medium from bone marrow-derived mesenchymal stem cells; Msx2: Msh homeobox 2; OC: Osteocalcin; rhBMP2: Human recombinant BMP-2; Runx2:- Runt-related transcription factor 2; VEGF: Vascular endothelial growth factor; VSMC: Vascular smooth muscle cell; $\beta$-GP: Beta-glycerophosphate}

\section{Funding \\ The research was supported by the grants from the Postdoctoral Fund of Zhejiang Province and Ningbo City, Zhejiang Provincial Program for Medicine and Health (2016KYB262; 2017KY575), and the Natural Science Foundation of Ningbo (2014A610269).}

\section{Availability of data and materials}

The datasets used and/or analyzed during the current study are available from the corresponding author on reasonable request.

\section{Authors' contributions}

WSS and HSW planned the study design, performed experiments, collected data, participated in statistical analysis, and drafted the manuscript. WJ, LYH, and ZRC carried out cell culture and cell staining, and performed molecular biology experiments. TMQ provided financial support and participated in manuscript writing. CHB critically revised the manuscript prior to submission. WN provided administrative support and helped in the analysis. CXM conceived the study and participated in the study design, financial support, and final approval of the manuscript. All authors read and approved the final manuscript.

Ethics approval and consent to participate Not applicable.

\section{Consent for publication}

All authors have agreed to the publication of this manuscript.

Competing interests

The authors declare that they have no competing interests.

\section{Publisher's Note}

Springer Nature remains neutral with regard to jurisdictional claims in published maps and institutional affiliations. 


\section{Author details}

'Department of Cardiology, Ningbo First Hospital, Ningbo 315000, China. ${ }^{2}$ Spine Tumor Center, Changzheng Hospital, Second Military Medical University, Shanghai 200003, China.

\section{Received: 17 October 2017 Revised: 17 April 2018}

Accepted: 2 May 2018 Published online: 13 June 2018

\section{References}

1. Vattikuti R, Towler DA. Osteogenic regulation of vascular calcification: an early perspective. Am J Physiol Endocrinol Metab. 2004;286(5):E686-96.

2. Abedin M, Tintut $Y$, Demer LL. Vascular calcification: mechanisms and clinical ramifications. Arterioscler Thromb Vasc Biol. 2004;24(7):1161-70.

3. Karwowski W, Naumnik B, Szczepański M, Myśliwiec M. The mechanism of vascular calcification - a systematic review. Med Sci Monit. 2012;18(1):RA111

4. Rennenberg RJ, Kessels AG, Schurgers LJ, Van Engelshoven JM, de Leeuw PW, Kroon AA. Vascular calcifications as a marker of increased cardiovascular risk: a meta-analysis. Vasc Health Risk Manag. 2009:5:185-97.

5. Chen NX, Duan D, O'Neill KD, Wolisi GO, Koczman JJ, Laclair R, et al. The mechanisms of uremic serum-induced expression of bone matrix proteins in bovine vascular smooth muscle cells. Kidney Int. 2006;70(6):1046-53.

6. Qiu C, Zheng H, Tao H, Yu W, Jiang X, Li A, et al. Vitamin K2 inhibits rat vascular smooth muscle cell calcification by restoring the Gas6/Axl/Akt antiapoptotic pathway. Mol Cell Biochem. 2017;433(1-2):149-59.

7. Wen C, Yang X, Yan Z, Zhao M, Yue X, Cheng X, et al. Nalp3 inflammasome is activated and required for vascular smooth muscle cell calcification. Int J Cardiol. 2013;168(3):2242-7.

8. Guo J, Lin GS, Bao CY, Hu ZM, Hu MY. Anti-inflammation role for mesenchymal stem cells transplantation in myocardial infarction. Inflammation. 2007:30(3-4):97-104

9. Oh JY, Kim MK, Shin MS, Lee HJ, Ko JH, Wee WR, et al. The antiinflammatory and anti-angiogenic role of mesenchymal stem cells in corneal wound healing following chemical injury. Stem Cells. 2008;26(4): 1047-55.

10. Ortiz LA, Dutreil M, Fattman C, Pandey AC, Torres G, Go K, et al. Interleukin 1 receptor antagonist mediates the antiinflammatory and antifibrotic effect of mesenchymal stem cells during lung injury. Proc Natl Acad Sci U S A. 2007; 104(26):11002-7.

11. Krabbe KS, Pedersen M, Bruunsgaard H. Inflammatory mediators in the elderly. Exp Gerontol. 2004;39(5):687-99.

12. Wang TJ, Larson MG, Levy D, Benjamin EJ, Kupka MJ, Manning WJ, et al. Creactive protein is associated with subclinical epicardial coronary calcification in men and women: the Framingham Heart Study. Circulation. 2002;106(10):1189-91.

13. Doherty TM, Fitzpatrick LA, Inoue D, Qiao JH, Fishbein MC, Detrano RC, et al Molecular, endocrine, and genetic mechanisms of arterial calcification. Endocr Rev. 2004;25(4):629-72.

14. Cai J, Pardali E, Sánchez-Duffhues G, ten Dijke P. BMP signaling in vascular diseases. FEBS Lett. 2012;586(14):1993-2002

15. Demer LL, Tintut Y. Vascular calcification: pathobiology of a multifaceted disease. Circulation. 2008;117(22):2938-48

16. Gupta N, Su X, Popov B, Lee JW, Serikov V, Matthay MA. Intrapulmonary delivery of bone marrow-derived mesenchymal stem cells improves survival and attenuates endotoxin-induced acute lung injury in mice. J Immunol. 2007;179(3):1855-63

17. Lee RH, Pulin AA, Seo MJ, Kota DJ, Ylostalo J, Larson BL, et al. Intravenous hMSCs improve myocardial infarction in mice because cells embolized in lung are activated to secrete the anti-inflammatory protein TSG-6. Cell Stem Cell. 2009;5(1):54-63.

18. Tögel F, Hu Z, Weiss K, Isaac J, Lange C, Westenfelder C. Administered mesenchymal stem cells protect against ischemic acute renal failure through differentiation-independent mechanisms. Am J Physiol Renal Physiol. 2005:289(1):F31-42.

19. Sheikh AM, Nagai A, Wakabayashi K, Narantuya D, Kobayashi S, Yamaguchi $S$, et al. Mesenchymal stem cell transplantation modulates neuroinflammation in focal cerebral ischemia: contribution of fractalkine and IL-5. Neurobiol Dis. 2011:41(3):717-24.

20. Lee JK, Jin HK, Endo S, Schuchman EH, Carter JE, Bae JS. Intracerebral transplantation of bone marrow-derived mesenchymal stem cells reduces amyloid-beta deposition and rescues memory deficits in
Alzheimer's disease mice by modulation of immune responses. Stem Cells. 2010;28(2):329-43.

21. Yao L, Li ZR, Su WR, Li YP, Lin ML, Zhang WX, et al. Role of mesenchymal stem cells on cornea wound healing induced by acute alkali burn. PLoS One. 2012;7(2):e30842

22. Timmers L, Lim SK, Hoefer IE, Arslan F, Lai RC, van Oorschot AA, et al. Human mesenchymal stem cell-conditioned medium improves cardiac function following myocardial infarction. Stem Cell Res. 2011:6(3):206-14

23. Chen $Y X$, Zeng ZC, Sun J, Zeng HY, Huang Y, Zhang ZY. Mesenchymal stem cell-conditioned medium prevents radiation-induced liver injury by inhibiting inflammation and protecting sinusoidal endothelial cells. J Radiat Res. 2015:56(4):700-8.

24. Tamari M, Nishino Y, Yamamoto N, Ueda M. Acceleration of wound healing with stem cell-derived growth factors. Int J Oral Maxillofac Implants. 2013; 28(6):e369-75

25. Kim HS, Shin TH, Lee BC, Yu KR, Seo Y, Lee S, et al. Human umbilical cord blood mesenchymal stem cells reduce colitis in mice by activating NOD2 signaling to COX2. Gastroenterology. 2013;145(6):1392-403.e1-8.

26. Ding HT, Wang CG, Zhang TL, Wang K. Fibronectin enhances in vitro vascular calcification by promoting osteoblastic differentiation of vascular smooth muscle cells via ERK pathway. J Cell Biochem. 2006;99(5):1343-52.

27. Byon $\mathrm{CH}$, Javed A, Dai Q, Kappes JC, Clemens TL, Darley-Usmar VM, et al. Oxidative stress induces vascular calcification through modulation of the osteogenic transcription factor Runx2 by AKT signaling. J Biol Chem. 2008; 283(22):15319-27.

28. Sun Y, Byon CH, Yuan K, Chen J, Mao X, Heath JM, et al. Smooth muscle cell-specific runx2 deficiency inhibits vascular calcification. Circ Res. 2012; 111(5):543-52.

29. Hayashi K, Nakamura S, Nishida W, Sobue K. Bone morphogenetic proteininduced MSX1 and MSX2 inhibit myocardin-dependent smooth muscle gene transcription. Mol Cell Biol. 2006;26(24):9456-70.

30. Zhan JK, Wang YJ, Wang Y, Tang ZY, Tan P, Huang W, et al. The protective effect of GLP-1 analogue in arterial calcification through attenuating osteoblastic differentiation of human VSMCs. Int J Cardiol. 2015;189:188-93.

31. Derwall M, Malhotra R, Lai CS, Beppu Y, Aikawa E, Seehra JS, et al. Inhibition of bone morphogenetic protein signaling reduces vascular calcification and atherosclerosis. Arterioscler Thromb Vasc Biol. 2012;32(3):613-22.

32. Shimizu T, Tanaka T, Iso T, Matsui H, Ooyama Y, Kawai-Kowase K, et al. Notch signaling pathway enhances bone morphogenetic protein 2 (BMP2) responsiveness of Msx2 gene to induce osteogenic differentiation and mineralization of vascular smooth muscle cells. J Biol Chem. 2011:286(21):19138-48.

33. Tyson KL, Reynolds JL, McNair R, Zhang Q, Weissberg PL, Shanahan CM. Osteo/chondrocytic transcription factors and their target genes exhibit distinct patterns of expression in human arterial calcification. Arterioscler Thromb Vasc Biol. 2003;23(3):489-94.

34. Nakagawa Y, Ikeda K, Akakabe Y, Koide M, Uraoka M, Yutaka KT, et al. Paracrine osteogenic signals via bone morphogenetic protein-2 accelerate the atherosclerotic intimal calcification in vivo. Arterioscler Thromb Vasc Biol. 2010:30(10):1908-15.

35. Abe E, Yamamoto M, Taguchi Y, Lecka-Czernik B, O'Brien CA, Economides AN, et al. Essential requirement of BMPs-2/4 for both osteoblast and osteoclast formation in murine bone marrow cultures from adult mice: antagonism by noggin. J Bone Miner Res. 2000;15:663-73.

36. Shi $Y$, Massagué J. Mechanisms of TGF-beta signaling from cell membrane to the nucleus. Cell. 2003;113(6):685-700.

37. Wrana JL, Attisano L, Cárcamo J, Zentella A, Doody J, Laiho M, et al. TGF beta signals through a heteromeric protein kinase receptor complex. Cell. 1992:71(6):1003-14.

38. Zwijsen A, Verschueren K, Huylebroeck D. New intracellular components of bone morphogenetic protein/Smad signaling cascades. FEBS Lett. 2003; 546(1):133-9.

39. Attisano L, Lee-Hoeflich ST. The Smads. Genome Biol. 2001;2(8): REVIEWS3010.

40. Taylor IW, Wrana JL. SnapShot: The TGFbeta pathway interactome. Cell. 2008;133(2):378.e1.

41. Wan M, Cao X. BMP signaling in skeletal development. Biochem Biophys Res Commun. 2005;328(3):651-7.

42. Broege $A$, Pham $L$, Jensen ED, Emery A, Huang TH, Stemig M, et al. Bone morphogenetic proteins signal via SMAD and mitogen-activated protein 
(MAP) kinase pathways at distinct times during osteoclastogenesis. J Biol Chem. 2013;288(52):37230-40.

43. Wu X, Chim SM, Kuek V, Lim BS, Chow ST, Zhao J, et al. HtrA1 is upregulated during RANKL-induced osteoclastogenesis, and negatively regulates osteoblast differentiation and BMP2-induced Smad1/5/8, ERK and p38 phosphorylation. FEBS Lett. 2014;588(1):143-50.

44. Li X, Yang HY, Giachelli CM. BMP-2 promotes phosphate uptake, phenotypic modulation, and calcification of human vascular smooth muscle cells. Atherosclerosis. 2008;199(2):271-7.

- fast, convenient online submission

- thorough peer review by experienced researchers in your field

- rapid publication on acceptance

- support for research data, including large and complex data types

- gold Open Access which fosters wider collaboration and increased citations

- maximum visibility for your research: over $100 \mathrm{M}$ website views per year 